\title{
Cognitive Visual Perceptual Deficits in Delirium
}

L. J. E. Brown ${ }^{1,2}$, S. McGrory ${ }^{3}$, L. McLaren ${ }^{3}$, J. M. Starr ${ }^{1,2}$, I. J. Deary ${ }^{2,3}$, and

A. M. J. MacLullich ${ }^{1,2}$.

1: Geriatric Medicine Unit, University of Edinburgh, Edinburgh, U.K.

2: Centre for Cognitive Ageing and Cognitive Epidemiology, University of Edinburgh, Edinburgh, U.K.

3: Department of Psychology, University of Edinburgh, Edinburgh, U.K.

Corresponding Author: Dr Laura Brown.

Address: Department of Clinical and Surgical Sciences, Room S1642, Royal Infirmary of Edinburgh, 51 Little France Crescent, Edinburgh EH16 4SA, UK.

Email: laura.brown@ed.ac.uk

Tel: +44 (0) 1312426940

Fax: +44 (0) 1312426370

The Corresponding Author has the right to grant on behalf of all authors and does grant on behalf of all authors, an exclusive licence (or non exclusive for government employees) on a worldwide basis to the BMJ Publishing Group Ltd and its Licensees to permit this article (if accepted) to be published in the Journal of Neurology, Neurosurgery \& Psychiatry editions and any other BMJPGL products to exploit all subsidiary rights, as set out in our licence.

Keywords: Delirium; Cognition; Perception; Hallucinations; Memory; Word Count: 3173 


\section{ABSTRACT}

Background: Delirium is a neuropsychiatric disorder characterised by severe cognitive impairment, but its specific neuropsychological profile has not yet been clearly delineated. Psychiatric symptoms of perceptual disturbance, such as hallucinations, illusions and misperceptions, are also common in delirium, suggesting that patients may have deficits in the cognitive systems underlying visual perception. Methods: Five neuropsychological tests of visual perception were administered to 17 older patients with delirium, as well as to control groups of 14 patients with Alzheimer's dementia and 18 cognitively healthy controls. The Mini Mental State Examination (MMSE) and the CERAD verbal memory test were also administered to assess the specificity of any perceptual impairments.

Results: Patients with delirium scored significantly lower than cognitively normal controls on all perceptual tasks, and significantly lower than dementia patients on three of these tasks. MMSE scores did not differ between the delirium and dementia groups, and patients with delirium showed significantly better verbal recognition performance than the dementia group.

Conclusions: Patients with delirium have specific visual perceptual deficits that cannot be accounted for by general cognitive impairment. These novel findings provide insights into the neural mechanisms underling delirium, and may help to improve clinical detection and management of the disorder. The results also support previous suggestions that cognitive perceptual deficits play a causal role in eliciting psychiatric symptoms of perceptual disturbance. 


\section{INTRODUCTION}

Delirium (or "acute confusional state") is a severe neuropsychiatric disorder that is particularly prevalent in older, hospitalised patients.[1] The core diagnostic features of delirium are an acute onset and fluctuating deficits in attention and consciousness,[2] although several other cognitive and psychiatric symptoms are also associated with the disorder.[3] The psychopathology of delirium remains "remarkably understudied".[4] In particular, whilst marked cognitive impairment is known to occur in delirium,[5-10] detailed characterisation of its neuropsychological profile is lacking. Increasing our understanding of the particular cognitive domains that are affected in delirium may provide important insights into its underlying pathophysiological mechanisms.

One area of cognition that has not yet been examined in delirium is visual perception. This is surprising given that neuropsychiatric symptoms of perceptual disturbance, such as hallucinations, illusions and misperceptions, occur frequently in delirium.[3] However, most knowledge about the content and nature of the perceptual disturbances associated with delirium has come from subjective patient reports and clinical observations.[11] No published studies have yet been carried out to assess whether these patients also have specific cognitive deficits of visual perception.

Evidence that psychiatric perceptual disturbances may be associated with cognitive deficits of visual perception has come from other patients who frequently experience hallucinations, such as patients with Lewy body dementia (DLB)[12] and Parkinson's disease dementia (PDD).[13] These patients perform poorly on a range of cognitive 
tests of visual perception compared to patients with Alzheimer's dementia (AD) who are matched in terms of overall cognitive impairment but do not experience visual hallucinations.[14-17] Similarly, the frequencies of hallucinations associated with various types of dementia show strong positive correlations with the severity of their visual perceptual and attentional impairments, and yet do not correlate with the degree of their overall cognitive impairments.[18]

To account for these patterns, Collerton et al., (2005) proposed that these hallucinations may be caused by a combination of disturbed attentional processes and impoverished visual input. In their Perception and Attention Deficit (PAD) model,[18] they propose that the presence of both perceptual and attentional deficits results in incorrect and irrelevant neural representations of objects being activated in long-term memory by sensory inputs. The patient then experiences these erroneous cortical activations as hallucinations or illusory misperceptions.

As patients with delirium are known to show gross impairments of attentional functioning, $[3,5]$ and a high frequency of psychiatric perceptual disturbances, $[3,19]$ Collerton et al.'s model would predict that these patients also have cognitive deficits of visual perception. However, this hypothesis has not been formally tested. The aim of this study was therefore to measure the integrity of cognitive visual perceptual processing in patients with delirium using standard neuropsychological tests. The performance of these patients was compared to control groups of patients with AD or with no cognitive impairment. Additional tests of memory function and general cognitive impairment were also administered to all patients in order to ensure that any 
observed deficits of perceptual function in the delirium group could not be better accounted for by their degree of overall cognitive impairment. 


\section{METHODS}

\section{Participants}

Sixty two hospital patients provided written informed to consent to take part in the study, which was approved by the Scotland A Research Ethics Committee. Patients were in three groups: 1) patients with current delirium and no known dementia, 2) patients with $\mathrm{AD}$ and no current delirium, and 3) cognitively normal (control) patients with no known dementia and no current delirium. As both delirium and AD are associated with older age, only patients aged over 70 years were approached to take part. Exclusion criteria for all patients were visual or auditory impairments severe enough to affect the reliability of the cognitive testing.

Patients with delirium were recruited from acute medical and surgical wards of a general acute hospital. Potential participants were first identified by ward staff, who named eligible patients who had shown evidence of an acute change in cognitive function since being admitted. The presence of delirium was then formally assessed by one of the trained researchers (S.M. or L.M.) using the Confusion Assessment Method (CAM).[20] This diagnostic algorithm records the presence of the four core symptoms of delirium: 1) attentional impairment, 2) confused thinking, 3) disturbed consciousness, and 4) an acute onset or fluctuating course of symptoms. A diagnosis of delirium requires that symptoms 1 and 4 , and either 2 or 3 are present.

The CAM criteria were evaluated using a validated battery of measures.[21] This comprised the MMSE [22] and attentional assessments that required the patient to repeat strings of numbers in a forward or reverse order, and to list the days of the 
week and the months of the year backwards. The Delirium Symptom Interview [23] was also administered to most patients. Hospital staff and patients' medical notes provided additional diagnostic information. Five patients were subsequently excluded from the delirium group for showing insufficient symptomatic evidence to meet the full diagnostic criteria for delirium. It is possible that these patients did have delirium but that their symptoms were too subtle to have been detected at the time of examination. Alternatively, these patients may have had subsyndromal delirium, and/or another type of cognitive impairment that caused some of the clinical symptoms typical of delirium to be observed. One of these five patients also had a visual impairment that may have affected the validity of the perceptual tasks. One further patient was excluded for showing evidence of also having a pre-existing dementia, resulting in a final group size of 17.

Dementia patients were recruited from a hospital outpatient memory clinic. All dementia patients had been diagnosed by a geriatrician (J.S.) as having either AD or mixed AD and Vascular dementia according to ICD-10 criteria.[24] None of the 14 patients recruited into this group were subsequently excluded from the study.

Cognitively normal (control) patients were recruited from the same hospital wards as the patients with delirium. Patients were included if they had no evidence of dementia or delirium, as judged by the delirium assessments, their medical history, and their scores on the MMSE (using a cut-off score of 24/30). Seven patients were subsequently excluded from this group for not meeting these criteria. The final number of participants in this group was 18 . 


\section{Procedure}

The presence of delirium was first assessed in all participants using the assessment battery described previously. A second researcher (either L.M. or S.M.) then administered the cognitive tests, using the same fixed order for each participant.

Visual perception was tested using five tests taken from the Visual Object and Space Perception (VOSP) battery.[25] Space perception was measured using the dot counting and position discrimination tasks. In the dot counting task, participants are shown arrays of between five and nine randomly positioned black dots, and report how many dots they see each time. In the position discrimination task participants are shown two squares that each contains one black dot. The participant determines which of the two dots is positioned exactly in the centre of its square. Shape and object perception was tested using the shape detection, incomplete letters and object decision tasks. In the shape detection task participants determine whether each of 20 random speckled backgrounds contains a superimposed speckled X figure. In the incomplete letters task participants identify 20 capital letters, each of whose image has been degraded by randomly blanking out $70 \%$ of its surface. In the object decision task participants are shown silhouette images of one real object and three nonsense shapes on each trial, and they determine which image is of the real object. Participants are not required to name the objects. Previous research has shown that patients with Alzheimer's dementia are generally unimpaired on all of these tasks.[14-15, 26]

In order to evaluate the specificity of any perceptual deficits in the delirium group, verbal word memory was also assessed in each participant using the CERAD word learning test.[27] For this, participants are first shown 10 printed words, one at a time, 
and are asked to read each word aloud as it is presented. Immediate recall of the words is then tested by asking the participant to recall as many of the 10 words as they can. This procedure is repeated twice more, and the numbers of correctlyrecalled words are summed across the three trials to give a total immediate recall score out of 30. Delayed recall of the same words is then tested approximately 5-10 minutes later by again asking the participant to recall as many of the words as possible. This delayed recall score is calculated and expressed as a percentage of the participant's highest score of the three immediate recall trials. Delayed recognition is then tested by sequentially showing the participant the 10 target words intermixed with 10 new words, and asking them to report whether or not each word was from the original set. Performance on this task is measured by subtracting the number of false positive responses made by the patient from the number of words they correctly recognised to give a "recognition discrimination index" (RDI) value. This RDI value can therefore range from -10 to 10 , with higher scores being indicative of more accurate recognition.

All cognitive and delirium assessments were carried out in outpatient clinic rooms with the dementia patients, and on inpatient wards with the delirium and cognitively normal control patients. The testing environment of the dementia patients was therefore likely to have contained fewer distractions than that of the delirium and control group. However, as none of the perceptual tasks involved rapid stimulus presentations or required speeded responses, this difference in testing environment is unlikely to have affected the validity of the results. Furthermore, task instructions were repeated, and prompts to respond were given, whenever necessary during the testing sessions, thereby further minimising the effects of any external distractors. 


\section{Statistical Analyses}

Given that the distributions of most of the cognitive test data were non-normal, and variances were not always homogeneous between groups, non-parametric statistics were used to compare cognitive data between groups. Kruskal-Wallis tests were first used to look for overall differences among the three groups. Mann-Whitney U tests were then used to perform pairwise group comparisons. Statistical significance was taken as a two-sided $\mathrm{p}$ value $<.05$. 


\section{RESULTS}

\section{Patient Characteristics}

Demographic details of the participants in each group are shown in Table 1. Cognitively normal control patients were significantly younger than both delirium $(t(33)=2.35, \mathrm{p}<.05)$ and dementia patients $(t(30)=2.62, \mathrm{p}<.05)$. However there was no difference in age between the delirium and dementia groups $(t(29)=0.08, \mathrm{p}=$ .94). The median MMSE score of the control patients was higher than that of both the delirium $(U=3.0, \mathrm{p}<.001)$ and dementia patients $(U=15.0, \mathrm{p}<.001)$. However, MMSE scores of the delirium and dementia patients did not differ from one another $(U=99.5, \mathrm{p}=.44)$. The delirium and dementia patients were therefore similar in terms of age and overall level of cognitive impairment.

\begin{tabular}{|l|l|l|l|l|}
\hline & $\begin{array}{l}\text { Delirium } \\
\mathrm{n}=17\end{array}$ & $\begin{array}{l}\text { Dementia } \\
\mathrm{n}=14\end{array}$ & $\begin{array}{l}\text { Controls } \\
\mathrm{n}=18\end{array}$ & Between-groups \\
\hline Male:Female $^{1}$ & $6: 11$ & $7: 7$ & $5: 13$ & $\chi^{2}=1.70,2$ d.f., $\mathrm{p}=.43$ \\
\hline Age $^{2}$ (years) & $82.4 \pm 5.5$ & $82.5 \pm 4.3$ & $78.3 \pm 4.6$ & $\mathrm{~F}(2,46)=4.03, \mathrm{p}<.05$ \\
mean \pm SD & & 20.0 & 27.0 & $\mathrm{H}=29.90,2$ d.f., $\mathrm{p}<.001$ \\
\hline MMSE score & 20.0 & $(13-27)$ & $(24-30)$ & \\
\hline median (range) & $(9-25)$ & & & \\
\hline
\end{tabular}

Table 1: Demographic details of the patients in each group. Statistical comparisons between the three groups were performed using: chi square (1), analysis of variance (2), or Kruskal-Wallis (3) tests according to the type of data. 


\section{Visual Perception}

One patient in the delirium group was unable to complete the object decision task. All other perceptual tasks were completed by all participants.

Cognitively normal control patients generally scored well in all of the visual perceptual tasks (Fig. 1). At least $50 \%$ of these patients scored at, or close to, ceiling level in all tasks except for object decision. Even in this more difficult task, all control patients scored above chance level. These patterns are consistent with published norms for the tests.[25] Performance in the delirium and dementia groups was more variable (Fig. 1). Even so, very few patients in these groups scored at floor or below chance level in any of the tasks, showing that the difficulty level of the tasks was appropriate for the participants. Kruskal-Wallis tests revealed that there were significant differences among the three groups on all of these tests (dot counting: $\mathrm{H}=$ 12.32, 2 d.f., $\mathrm{p}<.01$; position discrimination: $\mathrm{H}=8.43,2$ d.f. $\mathrm{p}<.05$; shape detection: $\mathrm{H}=15.72,2$ d.f., $\mathrm{p}<.001$; incomplete letters: $\mathrm{H}=19.94,2$ d.f., $\mathrm{p}<.001$; object decision $\mathrm{H}=8.50,2$ d.f., $\mathrm{p}<.05)$.

Patients with delirium scored the lowest on all tests of visual perception. Their performance was significantly worse than control patients on all five of these tests (dot counting: median difference $=2.0, U=56.0, \mathrm{p}<.01$; position discrimination: median difference $=3.0, U=66.0, \mathrm{p}<.01 ;$ shape detection: median difference $=4.0$, $U=45.5, \mathrm{p}<.001 ;$ incomplete letters: median difference $=4.5, U=34.5, \mathrm{p}<.001 ;$ object decision: median difference $=5.0, U=65.5, \mathrm{p}<.01$ ). They also scored significantly lower than the dementia patients on the dot counting (median difference $=1.5, U=68.5, \mathrm{p}<.05)$, shape detection (median difference $=3.0, U=53.5, \mathrm{p}<.01)$, 
and incomplete letters (median difference $=4.0, U=35.0, \mathrm{p}<.001$ ) tasks. Their performance did not differ significantly from the dementia patients in the position discrimination (median difference $=2.0, U=90.0, \mathrm{p}=.26$ ) or the object decision task (median difference $=3.0, U=74.5, \mathrm{p}=.12$ ). Patients with dementia did not differ from the control group on any of these tasks (dot counting: median difference $=0.5, U$ $=95.5, \mathrm{p}=.25 ;$ position discrimination: median difference $=2.0, U=92.0, \mathrm{p}=.21$; shape detection: median difference $=1.0, U=87.0, \mathrm{p}=.15$; incomplete letters: median difference $=0.5, U=91.0, \mathrm{p}=.19 ;$ object decision: median difference $=2.0$, $U=84.5, \mathrm{p}=.12)$

\section{Insert Fig. 1 about here}

\section{Memory}

There were group differences on all three measures of memory performance (immediate recall: $\mathrm{H}=26.69,2$ d.f., $\mathrm{p}<.001$; delayed recall: $\mathrm{H}=25.80,2$ d.f., $\mathrm{p}<$ .001 ; delayed recognition: $\mathrm{H}=21.57,2$ d.f., $\mathrm{p}<.001)$. Patients with dementia had the lowest group scores on all three of these measures (Fig. 2). Their scores on all three measures were significantly lower than controls' (immediate recall: median difference $=9.5, U=12.5, \mathrm{p}<.001 ;$ delayed recall: median difference $=85.7 \%, U=9.0, \mathrm{p}<$ .001 ; delayed recognition: median difference $=5.5, U=14.5, \mathrm{p}<.001)$. Patients with delirium also scored lower than control patients on all three measures of memory (immediate recall: median difference $=6.5, U=19.0, \mathrm{p}<.001 ;$ delayed recall: median difference $=52.4 \%, U=43.0, \mathrm{p}<.001 ;$ delayed recognition: median difference $=3.5$, $U=60.5, \mathrm{p}<.01)$. However, the immediate recall scores of the delirium group did not differ from those of the dementia group (median difference $=3.0, U=102.0, \mathrm{p}=.52$ ). They also performed better than the dementia patients on the delayed recognition task 
(median difference $=2.0, U=67.5, \mathrm{p}<.05$ ), and showed a trend towards better performance on the delayed recall task (median difference $=33.3 \%, U=73.0, \mathrm{p}=$ $.07)$.

Insert Fig. 2 about here 


\section{DISCUSSION}

The main novel finding in this study is that patients with delirium were impaired relative to dementia and cognitively normal control patients on tests of visual perception. Importantly, patients with delirium showed a similar level of overall cognitive impairment and better memory performance than patients with dementia, suggesting that these findings are not due to generalised cognitive impairment. These results provide evidence that patients with delirium have specific cognitive deficits of visual perception, and contribute to our understanding of the neuropsychological profile of the disorder. These findings also parallel previous work showing similar perceptual deficits in DLB and PDD patients,[14-18] and are consistent with the suggestion that cognitive visual perceptual deficits are associated with psychiatric symptoms of perceptual disturbance.

The delirium group's poor performance on the perceptual tasks suggests that these patients have cognitive deficits in visual perceptual processing. However, it is not clear which elements of the perceptual system are affected. It may be that delirium is associated with direct impairments of basic visual processes in the occipital cortex, such as the determination of line orientation or spatial frequency analysis.[28] Alternatively, the perceptual deficits could result from impairments in the attentional systems responsible for directing processing resources to visual inputs. Attentional systems in the parietal lobe modulate neural activity in many areas of the occipital cortex that are involved in visual perceptual processing.[29-30] Under normal conditions this increases the sensitivity of perceptual judgements at attended locations.[31] However, attentional impairments in delirium may result in less 
effective directing or focusing of attention to appropriate aspects of visual stimuli during perception, thereby compromising the accuracy of perceptual judgements. Future studies that compare the extent of perceptual processing deficits across a wider range of visual and non-visual perceptual tasks may be able to uncover the functional mechanisms underlying patients' errors.

The results of this study parallel previously published data showing cognitive perceptual deficits in other neurological patients who experience psychiatric perceptual disturbances similar to those seen in delirium.[14-18] They are also consistent with the Perception and Attention Deficit model of hallucinations [18], which suggests that a combination of disturbed attentional processes and impoverished visual inputs play a causal role in eliciting hallucinations. In this study neither the frequency of patients' hallucinations nor the magnitude of their attentional impairments were measured, and so it was not possible to determine their association with the measured perceptual deficits. Nevertheless, as patients with delirium are already known to show attentional impairments, $[3,5]$ this new finding of cognitive visual perceptual deficits in delirium is consistent with the model's causal explanation. Further research is now needed to examine whether independent relationships can be found between the magnitude of cognitive perceptual and attentional deficits and the severity of psychiatric perceptual disturbances.

The findings of this study also have useful implications for the detection and clinical management of delirium. Diagnosing delirium can be difficult due to its considerable symptom overlap with other disorders commonly seen in the elderly population, such as the dementias. All but one of the perceptual tasks administered in this study were 
performed without difficulty by the majority of the cognitively normal control patients. $\mathrm{AD}$ patients also showed consistently higher performance than the delirium group in some of these tasks; most notably the shape detection and incomplete letters tasks. Incorporating items from these tests into diagnostic tools for delirium may therefore be useful in detecting delirium and in discriminating it from other disorders, enabling patients with delirium to be more rapidly identified and treated. This new evidence for visual perceptual problems in delirium may also help clinical care to be improved. For instance, the format of visual information given to patients could be made simpler and clearer, or even presented in a different sensory modality, so that patients are less likely to make perceptual errors, and so misunderstand the information.

In summary, these findings suggest that patients with delirium have specific cognitive deficits of visual perception that are not present in patients with Alzheimer's dementia. These results provide new information on the neuropsychology of delirium, and have implications for the assessment and management of this common, serious, and poorly-understood disorder. 


\section{ACKNOWLEDGEMENTS}

This work was funded by an MRC Clinician Scientist Fellowship to AM. The work was undertaken by The University of Edinburgh Centre for Cognitive Ageing and Cognitive Epidemiology, part of the cross council Lifelong Health and Wellbeing Initiative. Funding from the BBSRC, EPSRC, ESRC and MRC is gratefully acknowledged. We thank the patients and staff of the geriatric medicine and orthopaedics units of the Royal Infirmary of Edinburgh, and the memory clinic in the Royal Victoria Hospital, Edinburgh. 


\section{REFERENCES}

1. Siddiqi N, House AO, Holmes JD. Occurrence and outcome of delirium in medical in-patients: a systematic literature review. Age Ageing 2006;35:350-364.

2. American Psychiatric Association. Diagnostic and statistical manual of mental disorders, 4th edition, DSM-IV. Washington, DC: APA, 1994.

3. Meagher DJ, Moran M, Raju B, et al. Phenomenology of delirium: Assessment of 100 adult cases using standardised measures. Br J Psychiatry 2007;190:135-141.

4. Bhat R, Rockwood K. Delirium as a disorder of consciousness. J Neurol Neurosurg Psychiatry 2007;78:1167-1170.

5. O'Keeffe ST, Gosney MA. Assessing attentiveness in older hospital patients: global assessment versus tests of attention. J Am Geriatr Soc 1997;45:470-473.

6. Trzepacz PT, Baker RW, Greenhouse J. A symptom rating scale for delirium. Psychiatry Res 1988;23:89-97.

7. Christensen KJ, Bettin KM, Jilk KM, et al. Neuropsychological tests for monitoring delirium severity in elderly patients. Am J Geriatr Psychiatry 1994;4:6976. 
8. Wallesch CW, Hundsalz A. Language function in delirium: a comparison of single word processing in acute confusional states and probable Alzheimer's disease. Brain Lang 1994;46:592-606.

9. Hart RP, Levenson JL, Sessler CN, et al. Validation of a cognitive test for delirium in medical ICU patients. Psychosomatics 1996;37:533-546.

10. Chedru F, Geschwind N. Disorders of higher cortical functions in acute confusional states. Cortex 1972;8:395-411.

11. Lipowski ZJ. Delirium: Acute Confusional States. New York: Oxford University Press, 1990.

12. McKeith IG, Dickson DW, Lowe J, et al. Diagnosis and management of dementia with Lewy bodies: third report of the DLB Consortium. Neurology $2005 ; 65: 1863-1872$.

13. Papapetropoulos S, Katzen H, Schrag A, et al. A questionnaire-based (UMPDHQ) study of hallucinations in Parkinson's disease. BMC Neurol 2008;8:21.

14. Calderon J, Perry RJ, Erzinclioglu SW, et al. Perception, attention, and working memory are disproportionately impaired in dementia with Lewy bodies compared with Alzheimer's disease. J Neurol Neurosurg Psychiatry 2001;70:157-164. 
15. Lambon Ralph MA, Powell J, Howard D, et al. Semantic memory is impaired in both dementia with Lewy bodies and dementia of Alzheimer's type: a comparative neuropsychological study and literature review. J Neurol Neurosurg Psychiatry 2001;70:149-156.

16. Mori E, Shimomura T, Fujimori M, et al. Visuoperceptual impairment in dementia with Lewy bodies. Arch Neurol 2000;57:489-493.

17. Mosimann UP, Mather G, Wesnes KA, et al. Visual perception in Parkinson disease dementia and dementia with Lewy bodies. Neurology 2004;63:2091-2096.

18. Collerton D, Perry E, McKeith I. Why people see things that are not there: a novel Perception and Attention Deficit model for recurrent complex visual hallucinations. Behav Brain Sci 2005;28:737-757.

19. Gupta N, de Jonghe J, Schieveld J, et al. Delirium phenomenology: what can we learn from the symptoms of delirium? J Psychosom Res 2008;65:215-222.

20. Inouye SK, van Dyck $\mathrm{CH}$, Alessi CA, et al. Clarifying confusion: the confusion assessment method. A new method for detection of delirium. Ann Intern Med 1990;113:941-948.

21. Simon SE, Bergmann MA, Jones RN, et al. Reliability of a structured assessment for nonclinicians to detect delirium among new admissions to postacute care. J Am Med Dir Assoc 2006;77:412-415. 
22 Folstein MF, Folstein SE, McHugh PR. "Mini-mental state". A practical method for grading the cognitive state of patients for the clinician. $J$ Psychiatr Res $1975 ; 12: 189-198$.

23. Albert MS, Levkoff SE, Reilly C, et al. The delirium symptom interview: an interview for the detection of delirium symptoms in hospitalized patients. $J$ Geriatr Psychiatry Neurol 1992;5:14-21.

24. World Health Organisation. The ICD-10 classification of mental and behavioral disorders: clinical descriptions and diagnostic guidelines. Geneva: WHO Publications, 1992.

25. Warrington EK, James M. The Visual Object and Space Perception Battery. London: Harcourt Assessment, 1991.

26. Graham NL, Emery T, Hodges JR. Distinctive cognitive profiles in Alzheimer's disease and subcortical vascular dementia. J Neurol Neurosurg Psychiatry 2004;75:61-71.

27. Morris JC, Heyman A, Mohs RC, et al. The Consortium to Establish a Registry for Alzheimer's Disease (CERAD). Part I. Clinical and neuropsychological assessment of Alzheimer's disease. Neurology 1989;39:1159-1165.

28. Schiller PH. The central visual system. Vision Research 1986;1351-1386. 
29. Corbetta M, Miezin FM, Dobmeyer S, et al. Attentional modulation of neural processing of shape, color, and velocity in humans. Science 1990;248:1556-1559.

30. Somers DC, Dale AM, Seiffert AE, Tootell RB. Functional MRI reveals spatially specific attentional modulation in human primary visual cortex. Proc Natl Acad Sci U S A 1999;96:1663-1668.

31. Downing CJ. Expectancy and visual-spatial attention: effects on perceptual quality. J Exp Psychol Hum Percept Perform 1988;14:188-202. 


\section{Figure Captions}

Figure 1: Group results for each of the visual perceptual tasks. The interquartile range and median value of each dataset are represented by the height of the inner box and the position of the central horizontal line respectively. The positions of the upper and lower bars of each plot indicate the maximum and minimum non-outlier values of each dataset. Any outliers are represented by open circles on the plot. The position discrimination, object decision and shape detection tasks were all 2- or 4-alternative forced choice response tasks. Chance-level performance for each of these tasks is indicated on the plots with a dashed horizontal line. Symbols to the right of the median lines indicate group scores that differ significantly from cognitively healthy controls $(*)$ and dementia patients $(\dagger)$.

Figure 2: Group results for the immediate recall, delayed recall and delayed recognition measures of the verbal memory tasks. Symbols to the right of the median lines indicate group scores that differ significantly from cognitively healthy controls (*) and dementia patients $(\dagger)$. 

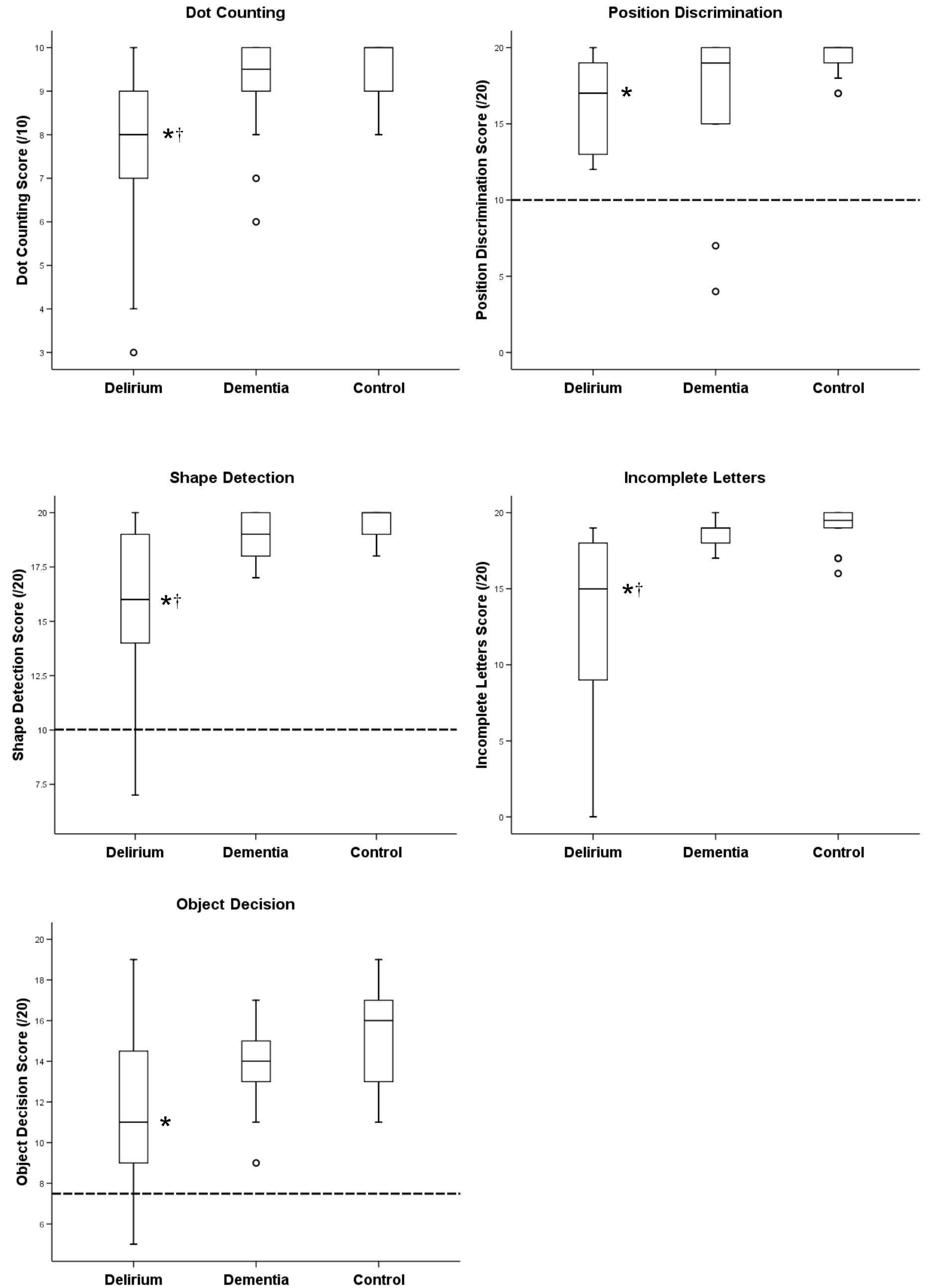

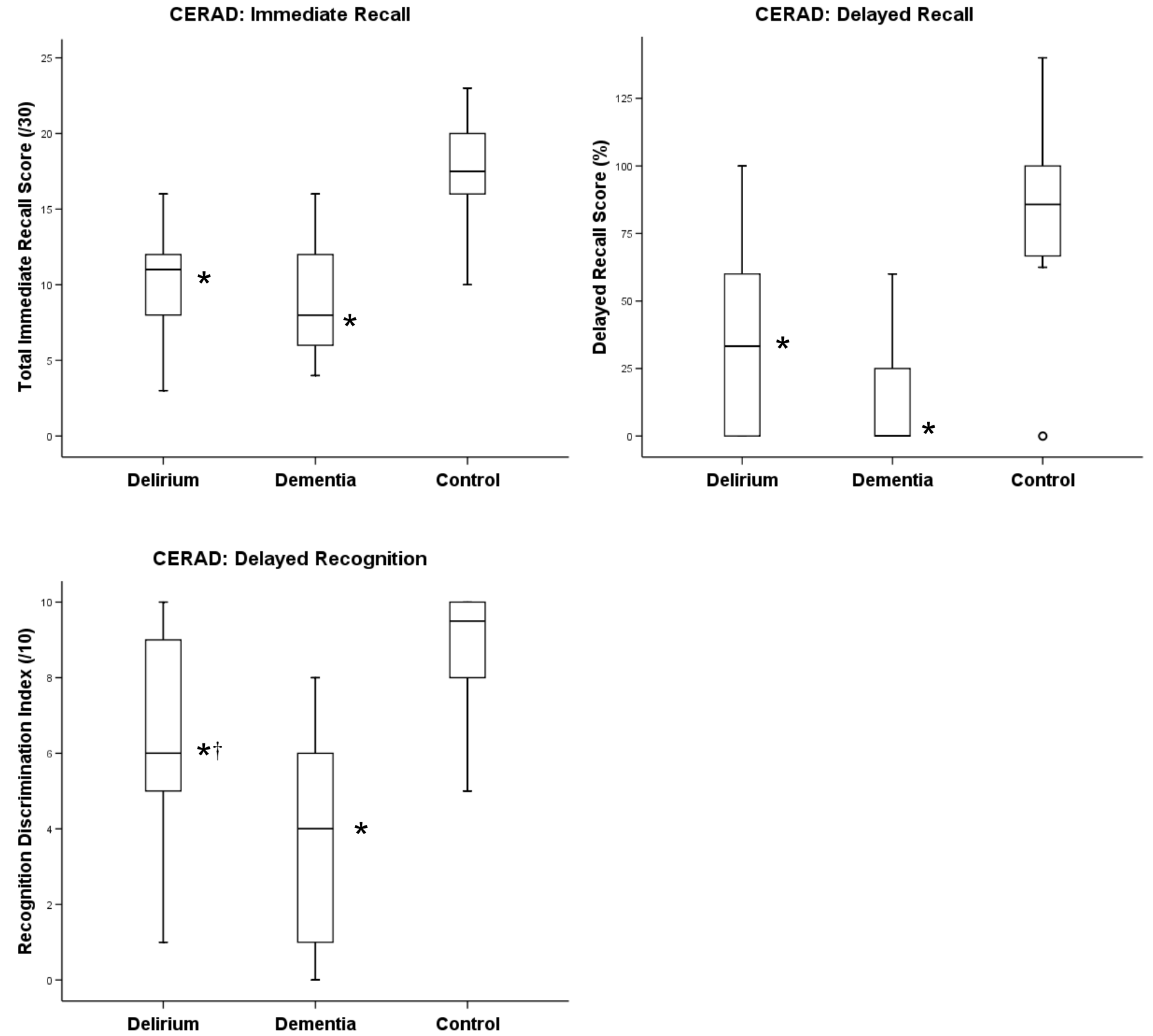